\title{
Drusen and pachydrusen: the definition, pathogenesis, and clinical significance
}

\author{
Xinyuan Zhang ${ }^{1} \cdot$ Sobha Sivaprasad $\mathbb{D}^{2}$
}

Received: 6 September 2020 / Revised: 20 October 2020 / Accepted: 28 October 2020 / Published online: 18 November 2020

(c) The Author(s), under exclusive licence to The Royal College of Ophthalmologists 2020

\begin{abstract}
The pachychoroid disease spectrum encompasses seven major retinal conditions including central serous chorioretinopathy (CSC), polypoidal choroidal vasculopathy (PCV), and pachychoroid neovasculopathy or type I macular neovascularisation (MNV) secondary to chronic persistent thickening and dysfunction of the choroidal vasculature. Drusen are focal yellow-white deposits of extracellular debris, which consist of complement proteins, esterified and nonesterified cholesterol, apolipoproteins, carbohydrates, and trace elements, above the retinal pigment epithelium (RPE) or between the RPE and Bruch's membrane. Although drusen are an essential disease precursor of advanced age-related macular degeneration (AMD), a new entity "pachydrusen" has been identified to be associated with some of the enitites that constitute the pachychoroid spectrum. It remains to be determined what the exact differences are between soft drusen, pseudodrusen, and pachydrusen in terms of phenotype, genotype, and pathogenesis. Improving our knowledge in these areas will inevitably improve our understanding of their clinical significance especially as in disease prediction in AMD and the pachychroid spectrum disorders. It remains controversial whether PCV is a subtype of AMD. Understanding the pathogenesis of different types of drusen may also help in addressing if phenotype and/or genotype of type $1 \mathrm{MNV}$ associated with pachychoroid are similar to type $1 \mathrm{MNV}$ related to AMD. Furthermore, because pachydrusen links two pachychoroid diseases, CSC and PCV, it is also of great interest to investigate if $\mathrm{CSC}$ is an early stage or a predictor of PCV in future research. In this review, we share our experience in clinical practice and the latest published evidence-based literature to emphasize the differences and similarities in morphology, pathogenesis, and clinical significance of drusen and pachydrusen, a new member of the pachychoroid spectrum disorders.
\end{abstract}

\section{Introduction}

The association between various drusen characteristics and the occurrence and progression of age-related macular degeneration (AMD) has been an area of research attention over the past three decades. In recent 5 years, the concept of pachychoroid disease spectrum (PCD) has been widely accepted since it was first proposed by David and colleagues in 2013 [1,2] with the advent of optical coherence tomography (OCT). Since then, the spectrum

Xinyuan Zhang

mmzxy2010@163.com

1 Beijing Institute of Ophthalmology, Beijing Tongren Eye Center, Beijing Tongren Hospital, Capital Medical University, Beijing 100730, PR China

2 NIHR Moorfields Biomedical Research Centre, Moorfields Eye Hospital, London, UK has diversified to include seven major diseases, including central serous chorioretinopathy (CSC), pachychoroid pigment epitheliopathy (PPE), pachychoroid neovasculopathy (PNV), polypoidal choroidal vasculopathy (PCV)/ aneurysmal type 1 neovascularization, focal choroidal excavation, and peripapillary pachychoroid syndrome. However, it is unclear whether PCD and AMD are the manifestations of the same condition or whether they are parallel diseases with some common features.

While drusen is thought to be diagnostic of AMD, the entity pachydrusen was recently coined by Rick Spaide in 2018 to describe drusenoid lesions, large size of $>125 \mu \mathrm{m}$ and solitary, in the context of a thickened choroid and distinct from the typical soft drusen of AMD [3]. Similar lesions have also been described by Bailey Freund as early as 2014 [4]. Both AMD and PCD are associated with yellow deposits in the fundus. The presence of reticular pseudodrusen or subretinal drusenoid deposits (SDDs) have added more complexity to the course of AMD and is usually associated with normal or thin choroid. Typical AMD-related drusen may be 
present in eyes with normal, thick, or thin choroid has been also observed. Thus, the correlation between drusen and PCD, especially with PCV and CSC, has become a new area of clinical and basic research.

In this review, Pubmed/MEDLINE, Springerlink, the Cochrane Library, Google Scholar, and EMbase Medline database engines were searched to retrieve English articles up to December 2019 with the terms "drusen", "pachydrusen", "pseudodrusen", "pachychoroid spectrum". The search was limited to "epidemiology" or "risk factors" or "pathogenesis"; or "imaging" or "trial" or "randomized" in order to mainly focus on the findings of more recent articles including randomized clinical trials, cohort study, meta-analyses, and reviews in the past decade.

\section{Discovery, classification, and morphology}

Understanding the pathway to discovery, morphology, and classification of drusen may help to further understand the clinical significance of different types of drusen (especially pachydrusen) and their predictive role in the development of diverse macular diseases (e.g., does the relative presence of drusen and pachydrusen in the fundus signify different phenotypes of AMD and PCV).

\section{Discovery of various extracellular deposits}

The yellow-white punctate deposits of drusen were first described and termed as "Colloidkugeln" (colloidal ball) in 1855 by Donders based on the pathological findings [5]. Anatomische further defined it "geode" [6] based on their yellow and shiny appearance under fundus ophthalmoscopy (German plural, meaning nodular-like yellow crystal extracellular debris inside the cavity; drusen in English). The Age-Related Eye Disease Study (AREDS) group classified drusen into three categories: hard, soft, and calcium according to the morphology and location based on the stereo color images. Drusen were further quantified by their location and size from C-0 to C-2 categories: C-0 [1/24 optic disc diameter, optic diameter (ODD)], C-1 (1/12 ODD), and C-2 (1/6 ODD) (corresponding to 63,125 , and $250 \mu \mathrm{m}$, respectively) as the standard circles. The morphology including the density, the edge, and/or the thickness of drusen can also be described on the fundus image.

In the early 20th century, subretinal extracellular drusenoid deposits were discovered, originally termed as "reticular pseudodrusen." They were first reported in 1990 by Mimoun et al. [7]. It was described as "pseudodrusen visible en lumière bleue (in French)" to define a new entity of deposits with diameter $>125 \mu \mathrm{m}$ with enhanced visibility in blue light [8]. Armold et al. defined these deposits as "reticular drusen" due to their pattern of "yellow interlacing network of $125-250 \mu \mathrm{m}$ wide appearing first in the superior outer macula and then extending circumferentially and beyond" [9]. The term "reticular drusen" or "reticular pseudodrusen" were then used in several prospective cohort studies, including Beaver Dam Study [10], Blue Mountains Eye Study, and Melbourne Collaborative Cohort studies [11, 12]. The Wisconsin Age-Related Maculopathy Grading System [13], applied the term "reticular drusen", which was also utilized for the Beaver Dam study [14], and AREDS [1517] to describe a group of drusen, which were initially thought to originate external to the retinal pigment epithelium (RPE), resembling typical drusen. In recent years, based on the evolving multimodal imaging platform, especially OCT, it was confirmed that these extracelluar accumulations of material are located internal to the RPE, that is, subretinal rather than the sub-RPE and so is now more commonly addressed as SDD [16, 18-21]. They have been reported in both AMD and PCD [22]. Based on multimodal imaging [color fundus images, near-infrared scanning laser ophthalmoscopic image, auto-fluorescence, and enhanced depth imaging OCT], Spaide and Curcio confirmed the location of three extracellular lesions, i.e., soft drusen, cuticular drusen, and SDDs in keeping with their histological location [23].

The entity "pachydrusen" was recognized only about 5 years ago. Deep phenotyping of retinal and choroidal diseases is made possible by the rapid recent innovation of fundus imaging system, especially OCT (significant improvements in both hardware and software capabilities). Swept source OCT (SS-OCT), a new generation OCT with higher speed and longer wavelength significantly improved the visualization of the retinal microvasculature and choriocapillaris and has helped us to understand the phenotype of PCD. By using multimodal imaging platform, pachydrusen are confirmed to be located in the posterior pole, beneath the RPE and described as isolated or scattered yellow-white deposits with welldefined boundary. Pachydrusen are found to be associated with thick subfoveal choroid by SS-OCT, further confirming that pachydrusen are a new entity distinct from soft drusen [1]. Subsequently the predictive role of pachydruen in disease progression has been established from nine publications from 2018 to 2020, confirming that pachydrusen are a precursor of PCV [24-32] and seven with CSC [27, 30, 33-36]. Pachydrusen are included as member of PCD spectrum because they are associated with common PCD features including reduced choroidal vascular markings in fundus and small pigment epithelium detachments due to the drusenoid RPE changes. The diagnostic criteria for PCD are mainly based on the changes in the structure and function of choroid, including 
Fig. 1 Fundus imaging of the drusen subtypes. A Pseudodrusen, B soft drusen, $\mathbf{C}$ pachydrusen.
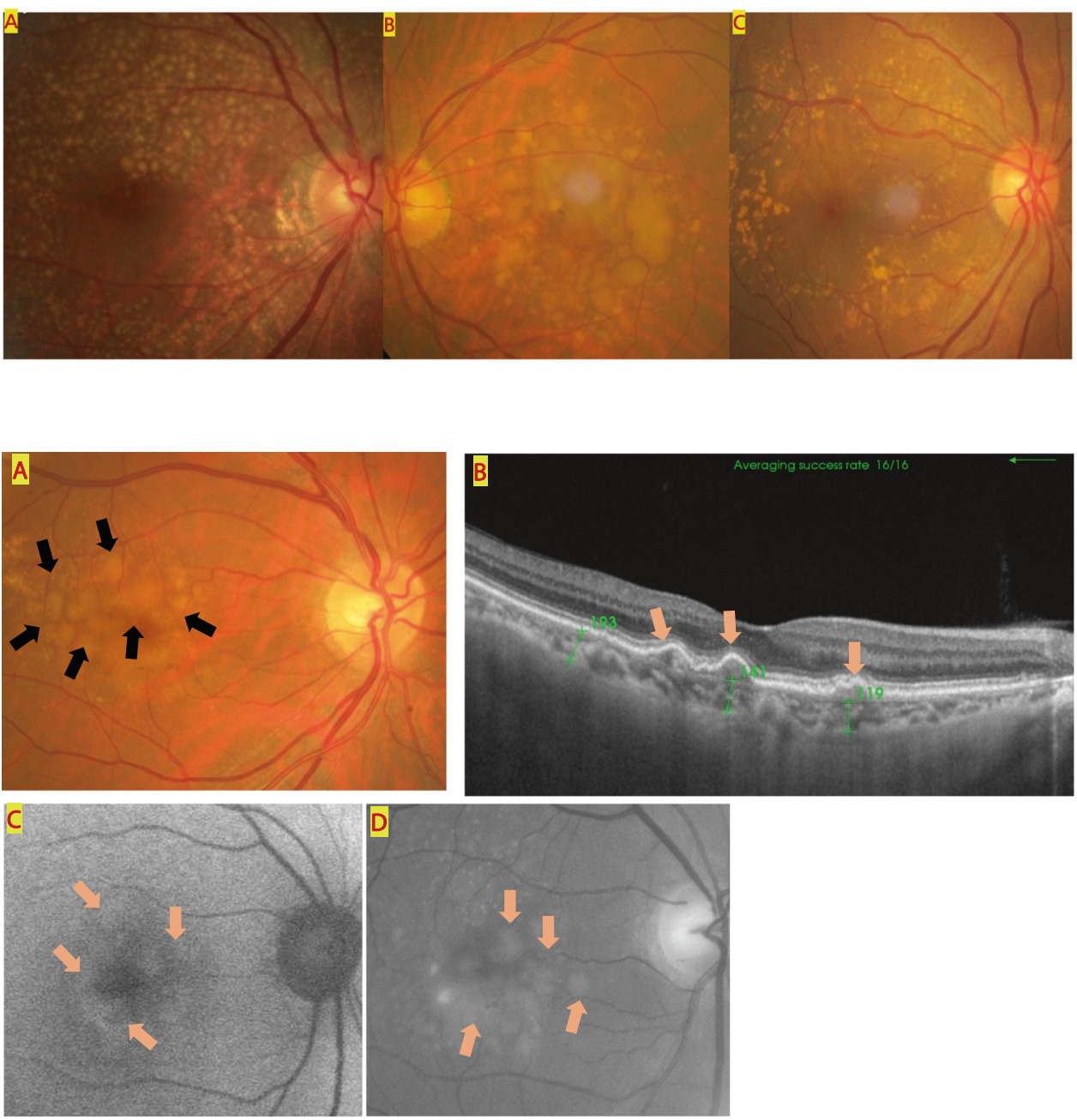

Fig. 2 Multimodality imaging of soft drusen. A A color fundus photography shows (black arrows) that the soft drusen appear as blurred boundary in the posterior pole with gradually reduced density from the center to the periphery of the retina. B OCT B scan shows (orange arrows) several semispherical elevation of retinal pigment epithelim, the subfoveal thickness of the choroid is $141 \mu \mathrm{m}$. C Hyperautoflorecence spots are detected correspondence with the findings of color fundus and infrared images (D) (orange arrows). OCT optical coherent tomography.
(1) increased choroidal thickness; (2) hypertrophy and dilatation of choroid vessels in the Haller layer; (3) hyperperfusion choroidal venous (hyperpermeability as detected by ICGA or dilated choroidal vessels as evidenced by en face OCT or OCTA); and (4) choroidal capillary atrophy and thinning of the vascular layer [37]. These pathological changes are characterized by chronic persistent choroidal thickening and choroidal dysfunction, unlike soft drusen, which is presumably a result of primary RPE-Bruch's membrane (BrM) abnormality. In contrast to both these yellow deposits, SDDs are located internal to the RPE and are associated with thin choroid. The multimodality imaging of soft drusen, reticular pseudodrusen, and pachydrusen is shown in Figs. 1-4.

\section{Classification and prevalence of different extracellular deposits}

The incidence of different types of extracellular deposits in AMD is variable, mainly due to the different techniques used to define the various subtypes of extracellular material in the outer retina and the age groups of the populations enrolled in different cohort studies. The Beaver Dam offspring study showed that the incidence of drusen is $63.3 \%$ of eyes in the cohort aged between 21 and 84 years. The frequency of drusen increased with age. The drusen $>125$ $\mu \mathrm{m}$ is presented in $0.6 \%$ of people aged between 21 and 34 years, but $9.2 \%$ in those aged $\geq 65$ years [38]. The occasional drusen detected among young people (age $<18$ years) are binocular. In Caucasians aged between 18 and 54 years, $91.48 \%$ of all gradable eyes had drusen within the central macular, drusen sized $<31.5 \mu \mathrm{m}$ presented in $89.7 \%$ of eyes, $>31.5$ and $<63 \mu \mathrm{m}$ in $45.9 \%$ of eyes, and $>63$ and $<125 \mu \mathrm{m}$ in $1.7 \%$ of eyes [39]. Other large-scale epidemiological cross-sectional studies based on fundus photography, e.g., the Chesapeake Bay study [40] (USA), the Rotterdam study (USA) [41], the Beaver Dam study [42] (USA), and Blue Mountain Eye study (Australia) [41] confirmed that the proportion of one or more drusen found in the fundus was 95.5-98.8\% in older population. The hard drusen with a diameter $<63 \mu \mathrm{m}$ are the most common type in all ages and gender. 


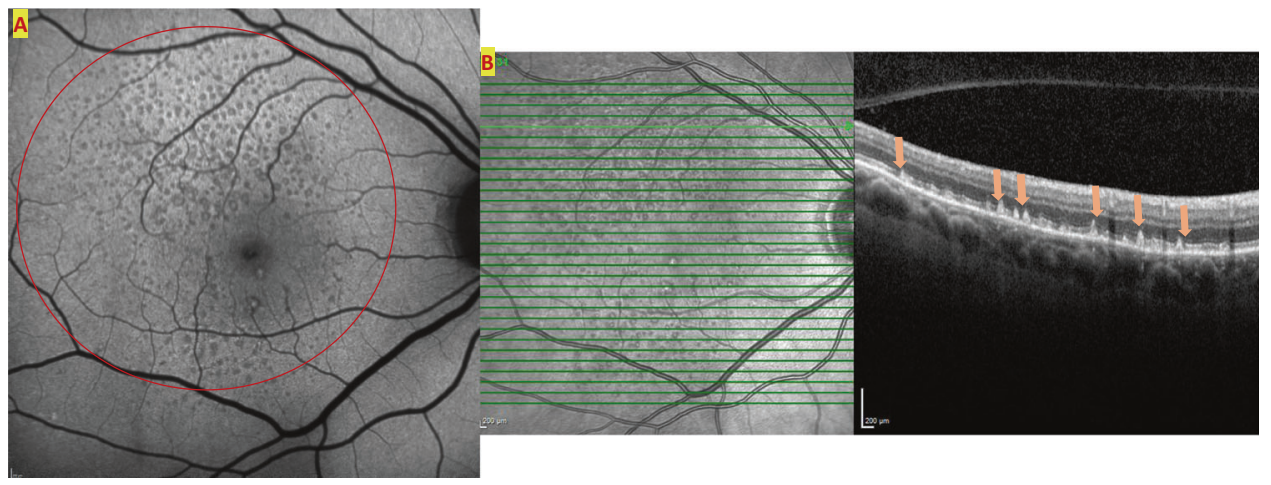

Fig. 3 Reticular pseudodrusen. A Reticular pseudodrusen are visualized as uniformly round deposits under blue light in the posterior pole (red cirle). B OCT B scan shows that reticular pseudodrusen, the extracelluar accumulations of material are located internal to the RPE, that is, subretinal rather than the sub-RPE and so is now more commonly addressed as subretinal drusenoid deposits (orange arrows). RPE retinal pigment epithelim. OCT optical coherent tomography.
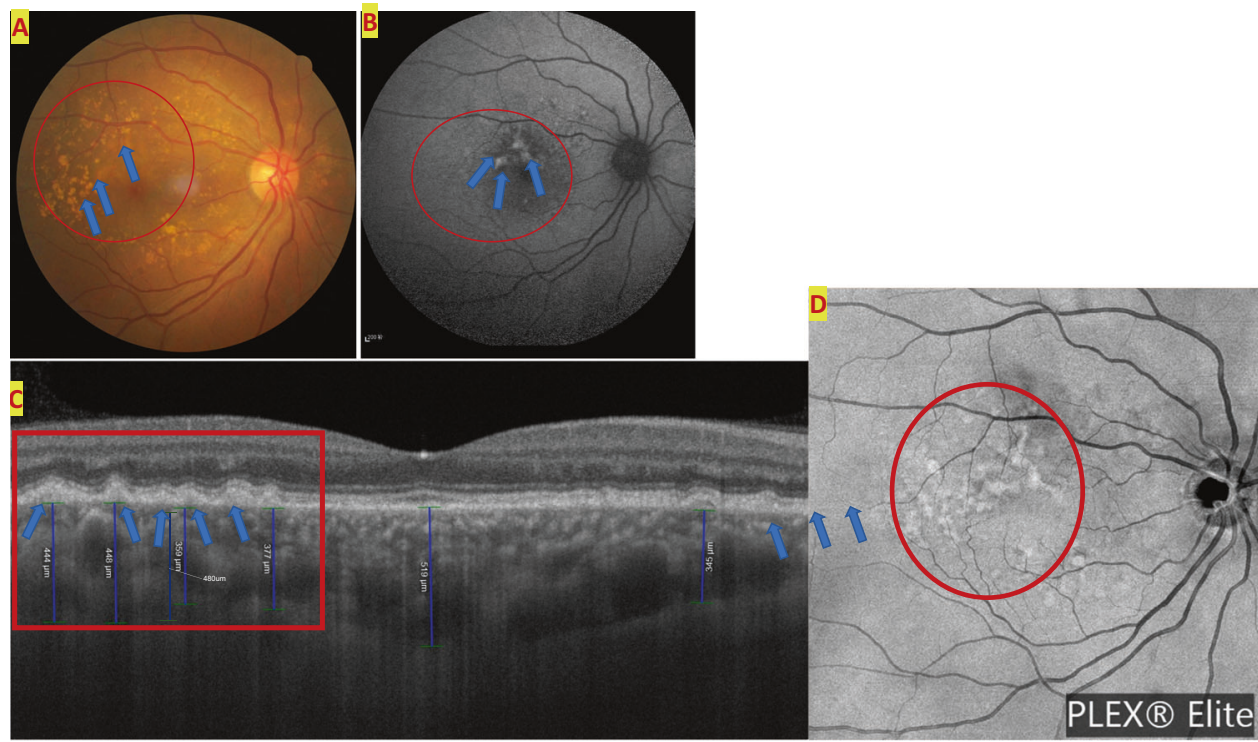

Fig. 4 Multimodality imaging of pachydrusen. A Color fundus photo showed yellowish scattered deposits in the posterior pole of the right eye (red circle and blue arrows). B Hyper-autoflorecence spots are detected correspondence with the findings of color fundus photo (red circle and blue arrows) in the same eye. C OCT B scan shows several high reflective deposits sub-RPE correspondent to the location

The prevalence of pseudodrusen is reported to be $0.41-1.95 \%$ and $3.0-4.0 \%$ detected by color fundus photography, which is much lower than the Rotterdam study (5\%) that utilize both color fundus photography and nearinfrared reflectance to confirm these lesions [43]. The prevalence is even higher (13.4-32\%) when multimodal imaging platform is used for diagnose [44, 45]. In addition, there appears to be racial predisposition in the incidence of pseudodrusen, with these deposit being more prevalent in older Caucasians and most cases are bilateral [46-48], compared to Asian populations [46]. with color fundus (blue arrows), the thickness under drusen and beneath the fovea is much higher than the normal eyes. D Pachydrusen are showed by en face OCT correspondence the location with color fundus photo of the same eye (blue arrows). RPE retinal pigment epithelim, OCT optical coherent tomography.

The prevalence of pachydrusen is $11.7 \%$ in a Caucasians cohort reported by Spaide, the mean subfoveal choroidal thickness (SFCT) was $419 \mu \mathrm{m}$ [49], while in an Indian cohort, the prevalence of pachydrusen is $8.4 \%$ with the mean SFCT $289.66 \mu$ [50]. In 2016, in an observational study on genotyping of AMD, the authors categorized 201 individuals into four groups: (1) neovascular AMD with presence of drusen, (2) PNV with type 1 choroidal neovascularisation, (3) thick choroid and absence or minimal presence of typical AMD drusen, and (4) absence of pachychoroid, whereas AMD or 
neovascularisation that served as the control group. Such study demonstrated that although individuals with PNV presented at a younger age compared to typical neovascular AMD, they shared several common risk alleles. On the contrary, the individuals with pachychoroid without neovasculopathy were of similar genetic disposition to the control group [51]. In 2017, with the advancement and development of OCT, Spaide [49] further investigated this specific group of PCD and observed that these eyes presented with drusen of size $>125 \mu \mathrm{m}$ in diameter but were distinctive from soft drusen in appearance, location, and aggregation; these lesions were termed as choroid-associated drusen or pachydrusen, which will be discussed below. The prevalence of pachydrusen in the treatment-naïve PCV is $70 \%$ reported by Lee and Byeon [24].

\section{Morphology of extracellular deposits}

Soft drusen usually appear as blurred boundary with gradually reduced density from the center to the periphery of the retina. The drusen that are not as solid, thick, nodular appearance are named hard drusen. Calcified drusen are defined as such when their surface is covered with white or shiny substance, which indicates calcium deposition [52]. They are predominantly seen in the posterior pole of the fundus. Pseudodrusen are yellow-white, uniformly round and such visibility could be enhanced in blue light. Pachydrusen often have a uniform appearance, they present as solitary, clustered, or isolated yellow-white deposits with a well-defined boundary.

Hard drusen is considered to be part of the normal aging process. A large number of soft drusen and retinal pigment abnormalities are well recognized as precursors for advanced AMD [53]. However, this field of research is evolving exponentially. For example, by using multimodal clinical imaging platform, hyporeflective internal reflectivity of drusen was found to be associated with disease progression in AMD in a retrospective cohort study $(>100$ patients), and a mineralomics analysis identified the composition in a small number of eyes shown to have this OCT phenotype [54]. On fundus photography, reticular pseudodrusen exist in only about 7-8\% of patients with any types of AMD, but about $21 \%$ geography (GA) of AMD eyes have pseudodrusen $[18,55]$. Pseudodrusen and soft drusen co-exist in patients with AMD and they have an initial predilection for the superior temporal part of the posterior pole and they disappear faster than soft drusen and their disappearance is often associated with outer retinal atrophy [56]. Despite different phenotypic presentations, both reticular pseudodrusen or SDD and soft drusen share several non-ocular risk factors e.g., older age $[10,57,58]$, female [57], smoking [10], and higher body index [12], suggesting a strong correlation between pseudodrusen and AMD [8]. Although there are limited reports on the course of pachydrusen, a recent literature showed that pachychoroid is linked to the development of MNV, especially type 2 and 3 MNV and geographical atrophy [56].

\section{Histopathology}

There is comparatively very scanty literature on histopathology of pseudodrusen and pachydrusen compared to drusen. Therefore, a review of histopathology of drusen may open up avenues of research comparing these extracellular deposits, which may provide insights into the various phenotypic presentations.

Age-related dysfunction of the RPE-BrM-choroid complex, together with inflammation, immune reaction, impaired cholesterol metabolism, and changes in extracellular matrix (ECM) are all considered as risk factors for the formation drusen and play a major role in its development and progression of AMD. Primary dysfunction of the RPE itself is also a critical primary pathologic feature in AMD (Fig. 5). RPE secretes proteins that are toxic to the outer retina and has a regulatory effect on the function of the BrM [59].

The BrM, the innermost layer of the choroid, is a unique pentalaminar structure and specific ECM located between the RPE and the fenestrated choroicapillaris. According to the classification of Hogan in the 1960s [60], BrM composes of distinct five layers (from inside to outside): the basement membrane of the RPE, the inner collagenous layer (ICL), the elastic layer (EL), the outer collagenous layer (OCL), and the choriocapillaris endothelium basement membrane. This anatomical compartmentalization is vitally important for the function of BrM, reinforcing why BrM is one of the focal points for local and systemic risk factors for initial stages of AMD [61]. Gass and Sarks both recommended a 3-layer BrM (ICL, EL, OCL) to best understand AMD pathology [62, 63]. Referred to as an "optical biopsy," OCT imaging has good correspondence with the histological slides. So to a certain extent, the OCT manesfications of the outer retina can illustrate the pathogenic process of drusen formation. Histopathological studies found that normal photoreceptor cells are organized and neatly arranged, and the outer segments of photoreceptor cells are closely attached to the RPE. These are mirrored as the ellipsoid layer on OCT. BrM present as uniform thickness and is challenging to be detected easily even under the highest resolution of OCT because of its tight binding to RPE. In the early AMD eyes, the photoreceptor cells are irregularly arranged and disordered, and the RPE and $\mathrm{BrM}$ are distorted due to the presence of drusen. Under the pathological conditions, such as RPE detachment, BrM 

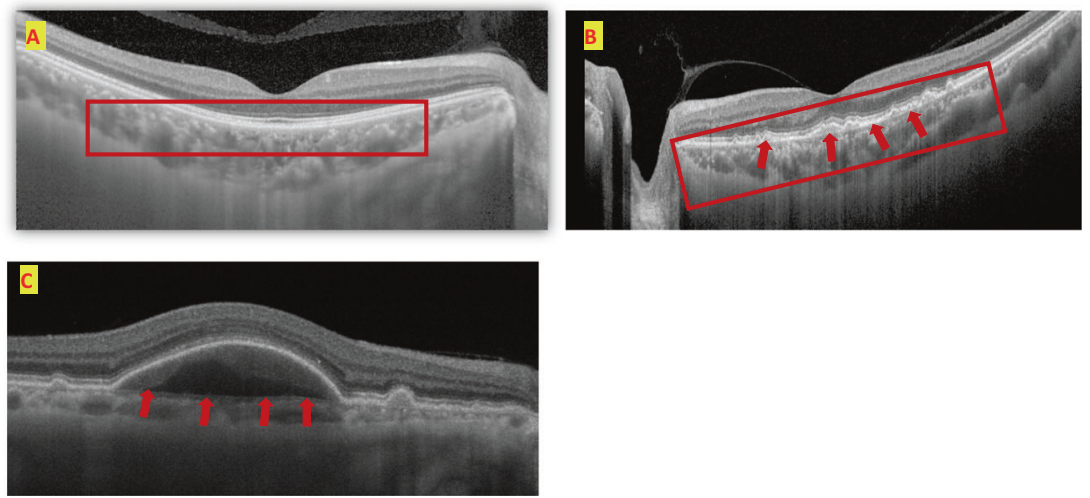

Fig. 5 The RPE-BrM-choroid complex. A In the normal eye (OCT B scan of right eye of a 66 years female, Plex Elite Zeiss) the BrM presented a uniform thickness and could not be detected easily even under the highest resolution of OCT because of its tight binding to RPE. B A 65 male complained gradual visual lose on his right eye for 2 years. The visual acuity of the eye is $20 / 60$. On OCT examination,
BrM separated from RPE (RPED) and could be visualized by OCT B scan (red arrows). C A 35 years male complained gradual visual lose and distortion on his left eye for 1 month. The visual acuity of the eye is 20/50. On OCT examination, BrM separated from RPE (RPED) and could be visualized by OCT B scan (red arrows). BrM Bruch membrane, RPED retinal pigment epithelium detachment.
A

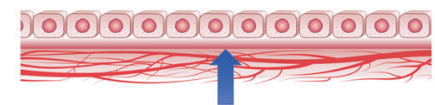

C

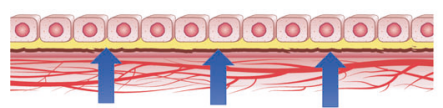

B

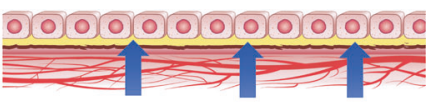

D

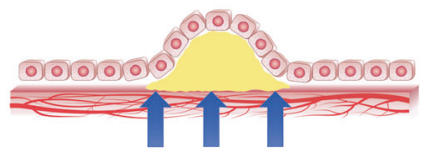

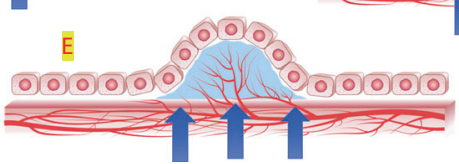

Fig. 6 Dysfunction of the RPE-BrM-choroid complex and formation of drusen. A The BrM presented a uniform thickness in the normal eye. B The deposits on the basement membrane can be classified into two broad categories based on the pathological characteristics: basal linear deposits (BLimD, the deposits on BrM). C The deposits within the membrane is defined as basal laminal deposits
(BLamD) (arrows). D Due to the accumulation of pathogenic proteins in BLmD and BLinD, the functional decompensation of the RPEBrM-choroid complex might induce the drusen formation. E Neovasuclarization as a consequence of drusen formation (arrows). BrM Bruch membrane, RPE retinal pigment epithelium. separates from RPE and is more easily observed on OCT B scan.

Although extracellular deposits external to the RPE increases with age, drusen, the extracellular deposits are typically associated with AMD. These deposits on the basement membrane can be classified into two broad categories based on the pathological characteristics. The deposits on BrM and between BrM and RPE are defined as a basal linear deposit (BLinD) and basal laminar deposit (BLamD), respectively. Green and Enger have proposed that BLamD and BLinD pose two dinstinct characteristics and positions relative to the RPE basal lamina (RPE-BL) $[64,65]$. BLamD is a critical phenotype in animal models and commonly used to study dry AMD [64]. BLamD is located between the RPE and RPE-BL, which contains of fibrous long-spacing collagen and can also be detected in the equatorial and peripheral regions of the retina [66]. On the other hand, BLinD is located between the RPE-BL and the ICL (i.e., external to the BLinD as encapsulated or unencapsulated membranous vesicle material localized in the inner colloidal layer of the BrM) $[65,67]$. It consists of membranous debris, which is also found in soft drusen $[65,68]$. BLinD, BLamD, and drusen contain ECM proteins, complement components, complement regulatory proteins, and inflammatory cytokines. These ECM proteins are also found in the deposits in the RPE layer [65]. The pathogical process of formation of BLinD, BLamD, and soft drusen is summazied in Figs. 6 and 7.

Most of the published work on drusen composition are based on drusen seen on color fundus photography that are 


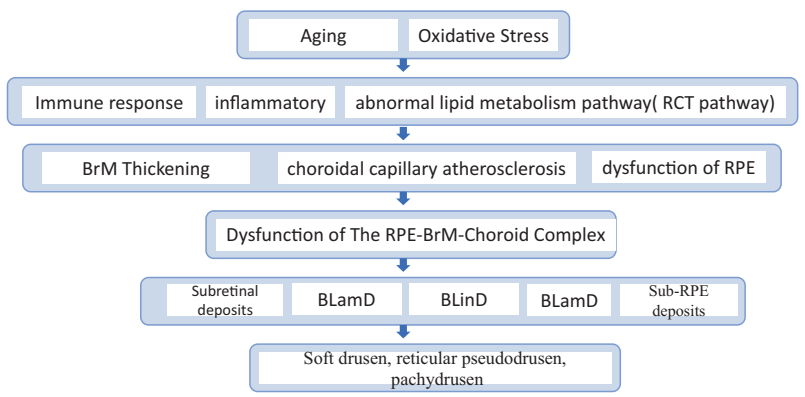

Fig. 7 Hypotheses on the pathogenesis and formation of drusen. Oxidative stress, inflammation, immunity response, cell and cell interaction, cell and cell-matrix adhesion, trigger the intracellular transport mechanism, inducing $\mathrm{BrM}$ thickening and dysfunction, decompensation of the RPE function, thickening of BrM, and atherosclerosis of choroidal capillaries. These pathological factors led to the formation of subretinal deposits, BLmD, BLinD, and sub-RPE deposits. Due to the accumulation of pathogenic proteins in BLamD and BLinD, the functional decompensation of the RPE-Bruch membrane-choroid complex might induce the drusen formation/choroidal neovascularization or polyps. RCT reverse cholesterol transport, BLamD basal laminal deposit, BLinD basal linear deposit RPE-BrMchoroid complex: retinal pigment epithelium-brunch membrane-choroid complex.

highly concentrated in the central macula $[69,70]$. One body of work that did focus on macula drusen found high concentration of lipids and evidence for expression of lipoprotein- and cholesterol-handling genes. Further, when considering the role of oxidative stress in AMD, a phototoxicity model is ideal although this has never borne out in epidemiology [71, 72] and does not account for any of the high-risk AMD biomarkers. In contrast, good evidence supports that soft drusen and SDDs relate topographically to cone and rod photoreceptors, respectively, implicating constitutive local mechanisms such as lipid transfer in deposit formation. In summary, based on histopathological findings, AMD is definitely a complex condition with interplay of multiple mechanisms including inflammation and immune response, abnormalities in intracellular transport mechanisms, BrM thickening, and dysfunction, as well as decompensation of the RPE function, and atherosclerosis of choroidal capillaries. These pathological factors contribute to the formation of BLmD and BLinD (the same material as soft drusen). Such pathological changes may cause functional decompensation of the RPE-Bruch membrane-choroid complex in different ways, leading to development of drusen formation/choroidal neovascularization or polyps [73] (Table 1).

Histopathological studies also revealed that the nodular drusen present as extracellular eosinophilic dome-shaped deposit [23]. Drusen material are strongly positive for periodic acid-Schiff staining, which indicates that high proportion of carbohydrate macromolecules (glycogen, glycoprotein, proteoglycans). The dense reaction of 
Periodic acid-Schiff stain in drusen also demonstrate the presence of mucopolysaccharides.

Furthermore, with increased accumulation of calcium, the basophilic staining gradually increases in drusen. Refractile drusen that appear as glistening reflective dots in the fundus correspond to heterogeneous internal reflectivity of drusen on OCT, and is positively stained by Von Kossa stain, indicating calcific granules or calcific stippling in the calcified drusen [54]. As the accumulation of basal or substrate linear deposits increases, local RPE detaches, and soft or large drusen can be found either between the RPE and its basement or outside the basement membrane of the RPE [64]. The size and density of drusen may predict disease progression [74].

Several trace elements are also detected in drusen, especially zinc [75, 76]. Zinc has a crucial role in normal retinal homeostasis [76] by regulating enzymes involved in the oxidative process [77]. Zinc supplements have been widely accepted as anti-oxidative agents to protect or delay the progression of AMD [22]. However, controversial opinion believes that the zinc contributes to the development of AMD. Based on the earlier findings that zinc contributes to deposit formation in neurodegenerative diseases including Alzheimer's and Parkinson's disease, Lengyel et al. demonstrated that sub-RPE deposits in postmortem human eye contain high level of zinc using a fluorescein-base probe for zinc (Zinpyr-1) [78]. This finding is further confirmed by detection of increased level of Zinc in the choroid with age, and in BrM of individuals with AMD especially at the macula [78]. The localization of zinc in AMD invites the hypothesis that zinc may be an early and crucial marker for drusen. Kuijk et al. reported that new and enhanced fluorescein-based probes for zinc, such as ZPP-1, is more effective than Zinpy-1 [79], suggesting that development of more sensitive in-vivo probe could improve our understanding of the pathogenesis of drusen formation and enhance research on the role of zinc in drusen formation and the early clinical diagnosis of AMD [79].

The source of protein and lipids in drusen remains unclear and might originate from both the RPE and choroid. However, systemic contribution cannot be ruled out [78]. The elements of the complement cascade seem to play a role in the pathogenesis of these extracellular deposits and provide evidence that immune response is involved in the occurrence and development of drusen [78]; for instance, complement factor $\mathrm{H}$, which plays a major role in the pathogenesis of drusen, can effectively slow the progression of AMD [78]. Drusen inhibit oxygen and nutrition diffusion from choroid to RPE and photoreceptors as they are located between the RPE and the choriocapillaries [80]. Other studies have also confirmed that drusen and RPE share the same protein profiles such as ATP synthase subunit beta, scavenger receptor $\mathrm{B} 2$, and retinol dehydrogenase 5, apoE,
apoB, complement component 9, clusterin and vitronectin in human autopsy eyes [81-84].

The proteins in BrM include collagen I-VI, elastin, basement membrane protein, glycosidic mucin, and nestin. In addition to provide structural support, BrM plays an integral role in maintainace of photoreceptor and choroicapillaries metabolism through signal transduction and filtration [85]. Recent studies have retained this focus on the pathological changes in BrM and its role in the pathogenesis of AMD and PCV [86]. Furthermore, a total of 20 proteins, including the immune proteins, have been identified, suggesting that the formation of drusen is associated with inflammation and the immune response of RPE [87-89]. In animal models, the expression level of EFEMP1, thrombospondin 1, milkfat globule-EGF factor 8, and collagen VI proteins in the BrM was found to be elevated and associated with the function of the ECM as assessed by the protein quantification assay [90]. In 2002, it was identified 129 proteins in drusen in human specimens (18 normal eyes and 5 AMD eyes) by proteomics analysis [91]. The differentially expressed proteins are tissue inhibitors of metalloproteinase 3 , clusterin, vitronectin, serum albumin, and crystallins as compared to normal human samples. Sixty proteins were detected in the drusen of cynomolgus monkeys, of which, $50 \%$ were consistent with the protein in human drusen [92]. The protein associated with oxidative damage, carboxymethyllysine (confirmed activation of downstream VEGF expression [93]), and advanced glycosylation end products is also found in human [91]. To date, there is no histochemistry and pathological evidence to show if the component of pachydrusen is same or similar to that of soft drusen [32].

Marked localized accumulation of neutral lipids that bind Oil Red $\mathrm{O}$ that recognizes unsterifid cholesterol, esterified cholesterol, trglycerides, and free acids in BrM, with age in normal eyes, have been reported by several studies [94-97]. This pathological process increases the resistance of the BrM, further decreasing vital exchange between the RPE and chorriocapillaries [98, 99]. As a consequence of aging, impaired cholesterol metabolism accelerates drusen pathogenesis, this can be explained by multiple mechanisms: (1) lipids are a major component of drusen, first discovered by Wedl, Wolter, and Falls [100, 101] and have been confirmed by other researchers [95, 102]; (2) variants of cholesterol-related genes (CETP, ABCA1, ABCG1, LIPC, and $A P O E$ ) have been identified to be correlated with the pathogeneisis of AMD and drusen formation [103]; (3) impaired cholesterol efflux (reverse cholesterol transport pathway) due to aging accelerates tissue-specific macrophages-mediated inflammation and AMD progression [82, 83, 104]; (4) dysfunction of RPE (also due to lipofuscin accumulation and mitochondrial degeneration), thickening of $\mathrm{BrM}$, and atherosclerosis of choroidal capillaries are all 
the key contributors of drusen formation that are lipidmediated [81-83, 103, 105-107]; (5) the mechanism that underlines macular involvement may also depend on the neuroglial relations and xanthophyll delivery (together with cholesterol) that correlate with drusen formation with aging, as suggested by Curcio et al. [105]; and (6) in a soft drusen/ BLinD formation model, RPE-secreted apoB- or apoEcontaining lipoprotein is retained by a dysfunctional BrM, forming oily layers on the BrM surface, with oxidation or other modification to form BLinD [103].

Whilst there is significant evidence of the composition of drusen, there are less reports on reticular pseudodrusen or SDD. Zweifel graded these SDD internal to the RPE, based on SD-OCT, into four stages [108]. SDD and soft drusen were likely to be found in AMD eyes with normal or thinner choroid [49, 56, 109, 110]. Querques et al. found that frequency of the stage of SDD changes over time, suggesting that pseudodrusen are dynamic pathological structures and associated with the occurrence and development of AMD and outer retinal atrophy $[8,111]$. Unlike traditional drusen, pseudodrusen is more commonly associated with a decreased sensitivity in abnormal microperimetry and decreased dark adaptation [112]. However, the correlation between pseudodrusen and retinal function needs to be further evaluated. Several cohort studies have shown that pseudodrusen are associated with GA and type 2 and 3 $\mathrm{MNV}$, whereas pachydrusen is associated with type I MNV and PCV [109]. Frameshift mutation in the ARMS2 and the complement factor $\mathrm{H}$ genes can be found in the eye with and without SDD in AMD [8]. Moreover, SDD has been shown as a risk factor for MNV (type 2 and 3) and GA. Therefore, SDD is generally considered to be a predictor for the occurrence and development of AMD.

The pathogenesis of pachydrusen has not been fully elucidated, however, recent genetic studies have provided evidence that PCD is different from drusen-driven AMD. Some genetic studies showed that the risk frequency of ARMS2 A69S is significantly lower in AMD patients with pachychoid drusen than those patients with soft and pseudodrusen [32, 99]. Furthermore, the risk allele in ARMS2 A69S is also found less commonly in AMD patients with GA, which was diagnosed in $23 \%$ of the total study cohort than in the patients with conventional GA, implying AMD patients with pachychoid may have different clincial outcome in comparison with those with normal thickness of choroid [113]. A recent study has shown that $\mathrm{CFH}$ risk alleles, the established gene associated with development of the drusen and AMD, protect against pachychoroid development and pachychorid drusen formation [114]. Pachydrusen is usually found to have RPE abnormalities around the periphery, while large soft drusen may have hyperpigmentation on the surface. Pachydrusen is also found in dry AMD and correlated with thicker choroid and higher choroidal vascularity index, but the correlation between this clincial phenotype with the type $1 \mathrm{MNV}$ in neovascular AMD is not established [50]. Five major PCDs, including PCV, CSC, PPE, and type $1 \mathrm{MNV}$, were enrolled in 36 eyes with pachydrusen from 134 patients. The morphology of the choroid was analyzed using en face OCT. The study identified an increased choroidal thickness under the PCD, due to enlarged choroidal vessels in the Haller layer with atrophy of the choroidal capillaries [115]. Kang et al. found that soft drusen is closely related to the development of neovascular AMD, but alterations in the RPE are associated with PCV. The diameter of pseudodrusen is $>125 \mu \mathrm{m}$ due to the altered RPE [116]. In a retrospective case series of 302 eyes of 151 patients with treatment-naïve CSC, pachydrusen were found localized within the choriocapillaries geographic filling delay and over the dilated outer choroidal vessels, indicating that pachydrusen is an indicator of choroidal impairment and is a feature of chronic persistent or resolved CSC [33]. Whether pachydrusen is a precursor or a tell-tale sign of underlying pachychoroid phenotype is a new direction of research that has to be answered by large-scale populationbased studies. The clinical significance of pseudodrusen, pachydrusen, and soft drusen in the pathogenesis of neovascular AMD and PCV is summarized in Table 1.

In summary, the interpretation of pachydrusen is reliant on advances in multimodal imaging. Continuous progress in the area of pathology and molecular biology is required for a deeper understanding of the underlying mechanism of pachydrusen and its association with PCV. PCV is a severe blinding condition and so it is of great interest to interrogate whether CSC is an early stage of PCV. The literature in this topic is being continuously updated based on the understanding of the pathogenesis of PCD. Previous study has suggested that PCV may have two distinct phenotypes, PNV and drusen-driven PCV based on their different genotypes. Therefore, genetic viewpoints may also provide the evidence required to distinguish PCD and drusen-driven AMD. Furthermore, the discovery of pathogenesis of PCD will underlie the clinical discovery of new molecular biological markers and targets. There is an unmet need for clinical evidence from longitudinal cohort studies with large sample size to evaluate whether the course of pachydrusen influences the development of PCV or the spontaneous resolution of CSC.

Acknowledgements This work is supported by the National Natural Science Foundation of China [Grant 81570850; Grant 81170859] and the Ministry of Science and Technology Foundation of China [Grant 2016YFC1305604].

\section{Compliance with ethical standards}

Conflict of interest The authors declare that they have no conflict of interest. 
Publisher's note Springer Nature remains neutral with regard to jurisdictional claims in published maps and institutional affiliations.

\section{References}

1. Warrow DJ, Hoang QV, Freund KB. Pachychoroid pigment epitheliopathy. Retina. 2013;33:1659-72.

2. Pang CE, Freund KB. Pachychoroid neovasculopathy. Retina. 2015;35:1.

3. Gallego-Pinazo R, Dolz-Marco R, Gómez-Ulla F, Mrejen S, Freund KB. Pachychoroid diseases of the macula. Med Hypothesis Discov Innov Ophthalmol. 2014;3:111-5.

4. Kim JH, Chang YS, Kim JW, Lee TG, Kim CG. Prevalence of subtypes of reticular pseudodrusen in newly diagnosed exudative age-related macular degeneration and polypoidal choroidal vasculopathy in Korean patients. Retina. 2015;35:2604-12.

5. Donders FC. Beiträge zur pathologischen Anatomie des Auges. Graef Arch Clin Exp. 1855;1:106-18.

6. Anatomische HM. Beiträge zur ophthalmologie - untersuchungen über die Glashäute des Auges, insbesondere die Glaslamelle der Chorioidea und ihre senilen Veränderungen. Graefes Arch Clin Exp. 1856;2:1-69.

7. Mimoun G, Soubrane G, Coscas G. [Macular drusen]. J Franais Dophtalmologie. 1990;13:511-30.

8. Rabiolo A, Sacconi R, Cicinelli MV, Querques L, Bandello F, Querques G. Spotlight on reticular pseudodrusen. Clin Ophthalmol. 2017;11:1707-18.

9. Arnold JJ, Sarks SH, Killingsworth MC, Sarks JP. Reticular pseudodrusen. A risk factor in age-related maculopathy. Retina. 1995;15:183-91.

10. Klein R, Meuer SM, Knudtson MD, Iyengar SK, Klein BE. The epidemiology of retinal reticular drusen. Am J Ophthalmol. 2008;145:317-26.

11. Finger RP, Chong E, McGuinness MB, Robman LD, Aung KZ, Giles G, et al. Reticular pseudodrusen and their association with age-related macular degeneration: the melbourne collaborative cohort study. Ophthalmology. 2016;123:599-608.

12. Joachim N, Mitchell P, Rochtchina E, Tan AG, Wang JJ. Incidence and progression of reticular drusen in age-related macular degeneration: findings from an older Australian cohort. Ophthalmology. 2014;121:917-25.

13. Klein R, Klein BE, Knudtson MD, Meuer SM, Swift M, Gangnon RE. Fifteen-year cumulative incidence of age-related macular degeneration: the Beaver Dam Eye Study. Ophthalmology. 2007; 114:253-62.

14. Knudtson MD, Klein R, Klein BE. Physical activity and the 15year cumulative incidence of age-related macular degeneration: the Beaver Dam Eye Study. Br J Ophthalmol. 2006;90:1461-3.

15. Armstrong J, Davis M, Danis Jr R, Klein R, Klein B, Lee LY, et al. Reticular drusen as a baseline risk factor for progression to advanced AMD in AREDS. Invest Ophthalmol Vis Sci. 2005;46. --this is a conference (ARVO) Abstract.

16. Schmitz-Valckenberg S, Alten F, Steinberg JS, Jaffe GJ, Fleckenstein M, Mukesh BN, et al. Reticular drusen associated with geographic atrophy in age-related macular degeneration. Invest Ophthalmol Vis Sci. 2011;52:5009-15.

17. Gil JQ, Marques JP, Hogg R, Rosina C, Cachulo ML, Santos A, et al. Clinical features and long-term progression of reticular pseudodrusen in age-related macular degeneration: findings from a multicenter cohort. Eye. 2017;31:364-71.

18. Cohen SY, Dubois L, Tadayoni R, Delahaye-Mazza C, Debibie C, Quentel G. Prevalence of reticular pseudodrusen in agerelated macular degeneration with newly diagnosed choroidal neovascularisation. Br J Ophthalmol. 2007;91:354-9.
19. Ueda-Arakawa N, Ooto S, Nakata I, Yamashiro K, Tsujikawa A, Oishi A, et al. Prevalence and genomic association of reticular pseudodrusen in age-related macular degeneration. Am J Ophthalmol. 2013;155:260-269.e2.

20. Hogg RE, Silva R, Staurenghi G, Murphy G, Santos AR, Rosina $\mathrm{C}$, et al. Clinical characteristics of reticular pseudodrusen in the fellow eye of patients with unilateral neovascular age-related macular degeneration. Ophthalmology. 2014;121:1748-55.

21. Naysan J, Jung JJ, Dansingani KK, Balaratnasingam C, Freund KB. Type 2 (subretinal) neovascularization in age-related macular degeneration associated with pure reticular pseudodrusen phenotype. Retina. 2016;36:449-57.

22. Age-Related Eye Disease Study Research Group. A randomized, placebo-controlled, clinical trial of high-dose supplementation with vitamins $\mathrm{C}$ and $\mathrm{E}$, beta carotene, and zinc for age-related macular degeneration and vision loss: AREDS report no. 8. Arch Ophthalmol. 2001;119:1417-36.

23. Spaide RF, Curcio CA. Drusen characterization with multimodal imaging. Retina. 2010;30:1441-54.

24. Lee J, Byeon SH. Prevalence and clinical characteristics of pachydrusen in polypoidal choroidal vasculopathy: multimodal image study. Retina. 2019;39:670-8.

25. Fukuda Y, Sakurada Y, Sugiyama A, Yoneyama S, Matsubara M, Kikushima W, et al. Title: Pachydrusen in fellow eyes predict response to aflibercept monotherapy in patients with polypoidal choroidal vasculopathy. J Clin Med. J Clin Med. 2020;9:2459. https://doi.org/10.3390/jcm9082459.

26. Matsubara M, Sakurada Y, Sugiyama A, Fukuda Y, Parikh R, Kashiwagi K. Response to photodynamic therapy combined with intravitreal aflibercept for polypoidal choroidal vasculopathy depending on fellow-eye condition:2-year results. PLoS ONE. 2020;15:e237330.

27. Yanagi Y. Pachychoroid disease: a new perspective on exudative maculopathy. Jpn J Ophthalmol. 2020;64:323-37.

28. Lee J, Kim M, Lee CS, Kim SS, Koh HJ, Lee SC, et al. Drusen subtypes and choroidal characteristics in Asian eyes with typical neovascular age-related macular degeneration. Retina. 2020;40: 490-8.

29. Cheung CMG, Gan A, Yanagi Y, Wong TY, Spaide R. Association between choroidal thickness and drusen subtypes in age-related macular degeneration. Opthalmol Retina. 2018;2:1196-205.

30. Yamashiro K, Hosoda Y, Miyake M, Ooto S, Tsujikawa A. Characteristics of pachychoroid diseases and age-related macular degeneration: multimodal imaging and genetic backgrounds. J Clin Med. 2020;9:2034. https://doi.org/10.3390/jcm9072034.

31. Singh SR, Chakurkar R, Goud A, Rasheed MA, Vupparaboina KK, Chhablani J. Pachydrusen in polypoidal choroidal vasculopathy in an Indian cohort. Indian J Ophthalmol. 2019;67:1121-6.

32. Fukuda Y, Sakurada Y, Yoneyama S, Kikushima W, Sugiyama $\mathrm{A}$, Matsubara $\mathrm{M}$, et al. Clinical and genetic characteristics of pachydrusen in patients with exudative age-related macular degeneration. Sci Rep. 2019;9:11906.

33. Matsumoto H, Mukai R, Morimoto M, Tokui S, Kishi S, Akiyama H. Clinical characteristics of pachydrusen in central serous chorioretinopathy. Graefes Arch Clin Exp. 2019;257: 1127-32.

34. Singh SR, Chakurkar R, Goud A, Chhablani J. Low incidence of pachydrusen in central serous chorioretinopathy in an Indian cohort. Indian J Ophthalmol. 2020;68:118-22.

35. Takahashi A HY, Miyake M, Oishi A, Ooto S, Tsujikawa A. Clinical and genetic characteristic of pachydrusen in eyes with central serous chorioretinopathy and normal subjects. Invest Ophthalmol Vis Sci. 2019;60.5005. (ARVO abstract).

36. Sartini F, Figus M, Casini G, Nardi M, Posarelli C. Pachychoroid neovasculopathy: a type-1 choroidal neovascularization belonging to the pachychoroid spectrum-pathogenesis, imaging and 
available treatment options. International Ophthalmology. 2020;30. https://doi.org/10.1007/s10792-020-01522-1.

37. Zhang X, Lai TYY. Paying attention to the concept and research of pachychoroid disease spectrum. Chin J Exp Ophthalmol. 2017;35:385-90.

38. Klein R, Cruickshanks KJ, Nash SD, Krantz EM, Nieto FJ, Huang GH, et al. The prevalence of age-related macular degeneration and associated risk factors. Arch Ophthalmol. 2010;128:750-8.

39. Silvestri G, Williams MA, McAuley C, Oakes K, Sillery E, Henderson DC, et al. Drusen prevalence and pigmentary changes in Caucasians aged 18-54 years. Eye. 2012;26:1357-62.

40. Bressler NM, Bressler SB, West SK, Fine SL, Taylor HR. The grading and prevalence of macular degeneration in Chesapeake Bay watermen. Arch Ophthalmol. 1989;107:847-52.

41. Mitchell P, Smith W, Attebo K, Wang JJ. Prevalence of agerelated maculopathy in Australia. The Blue Mountains Eye Study. Ophthalmology. 1995;102:1450-60.

42. Klein R, Klein BE, Linton KL. Prevalence of age-related maculopathy. The Beaver Dam Eye Study. Ophthalmology. 1992;99:933-43.

43. Buitendijk GH, Hooghart AJ, Brussee C, de Jong PT, Hofman A, Vingerling JR, et al. Epidemiology of reticular pseudodrusen in age-related macular degeneration: the Rotterdam Study. Invest Ophthalmol Vis Sci. 2016;57:5593-601.

44. Chan H, Cougnard-Grégoire A, Delyfer M-N, Combillet F, Rougier M-B, Schweitzer C, et al. Multimodal imaging of reticular pseudodrusen in a population-based setting: the Alienor Study. Invest Ophthalmol Vis Sci. 2016;57:3058-65.

45. Zarubina AV, Neely DC, Clark ME, Huisingh CE, Samuels BC, Zhang Y, et al. Prevalence of subretinal drusenoid deposits in older persons with and without age-related macular degeneration, by multimodal imaging. Ophthalmology. 2016;123:1090-100.

46. Alten F, Clemens CR, Milojcic C, Eter N. Subretinal drusenoid deposits associated with pigment epithelium detachment in agerelated macular degeneration. Retina. 2012;32:1727-32.

47. Sohrab MA, Smith RT, Salehi-Had H, Sadda SR, Fawzi AA. Image registration and multimodal imaging of reticular pseudodrusen. Invest Ophthalmol Vis Sci. 2011;52:5743-8.

48. Smith RT, Sohrab MA, Busuioc M, Barile G. Reticular macular disease. Am J Ophthalmol. 2009;148:733-43.e2.

49. Spaide RF. Disease expression in nonexudative age-related macular degeneration varies with choroidal thickness. Retina. 2018;38:708-16.

50. Singh SR, Oli A, Mohan S, Goud A, Rasheed MA, Vupparaboina KK, et al. Pachydrusen in Indian population: a hospitalbased study. Indian J Ophthalmol. 2019;67:371-5.

51. Dansingani KK, Perlee LT, Hamon S, Lee M, Shah VP, Spaide RF, et al. Risk alleles associated with neovascularization in a pachychoroid phenotype. Ophthalmology. 2016;123:2628-30.

52. Age-Related Eye Disease Study Research Group. The AgeRelated Eye Disease Study system for classifying age-related macular degeneration from stereoscopic color fundus photographs: the Age-Related Eye Disease Study report number 6. Am J Ophthalmol. 2001;132:668-81.

53. Uchida A, Srivastava SK, Manjunath D, Singh RP, Rachitskaya $\mathrm{AV}$, Kaiser PK, et al. Impact of drusen burden on incidence of subclinical MNV with OCTA. Opthalmic Surg Lasers Imaging Retina. 2019;51:22-30.

54. Tan AC, Pilgrim MG, Fearn S, Bertazzo S, Tsolaki E, Morrell AP, et al. Calcified nodules in retinal drusen are associated with disease progression in age-related macular degeneration. Sci Transl Med. 2018;10:eaat4544. https://doi.org/10.1126/scitra nslmed.aat 4544 .
55. Klein R, Meuer SM, Knudtson MD, Iyengar SK, Klein BE. The epidemiology of retinal reticular drusen. Am J Ophthalmol. 2008;145:317-26.

56. Switzer DW Jr, MendonÇa LS, Saito M, Zweifel SA, Spaide RF. Segregation of ophthalmoscopic characteristics according to choroidal thickness in patients with early age-related macular degeneration. Retina. 2012;32:1265-71.

57. Boddu S, Lee MD, Marsiglia M, Marmor M, Freund KB, Smith RT. Risk factors associated with reticular pseudodrusen versus large soft drusen. Am J Ophthalmol. 2014;157: 985-993.e2.

58. Wilde C, Patel M, Lakshmanan A, Morales MA, Dhar-Munshi S, Amoaku WM. Prevalence of reticular pseudodrusen in eyes with newly presenting neovascular age-related macular degeneration. Eur J Ophthalmol. 2016;26:128-34.

59. Hageman GS, Mullins RF. Molecular composition of drusen as related to substructural phenotype. Mol Vis. 1999;5:28.

60. Hogan MJ, Alvarado J. Studies on the human macula. IV. Aging changes in Bruch's membrane. Arch Ophthalmol. 1967;77: 410-20.

61. Booij JC, Baas DC, Beisekeeva J, Gorgels TG, Bergen AA. The dynamic nature of Bruch's membrane. Prog Retin Eye Res. 2010;29:1-18.

62. Bressler SB BN, Sarks SH, Sarks JP. Age-related macular degeneration: nonneovascular early AMD, intermediate AMD, and geographic atrophy. 4th ed. Louis: Mosby; 2006.

63. Gass JDM. Stereoscopic atlas of macular diseases: diagnosis and treatment. 4th ed. Louis: Mosby; 1997.

64. Green WR, Enger C. Age-related macular degeneration histopathologic studies. The 1992 Lorenz E. Zimmerman Lecture. Ophthalmology. 1993;100:1519-35.

65. Curcio CA, Millican CL. Basal linear deposit and large drusen are specific for early age-related maculopathy. Arch Ophthalmol. 1999;117:329-39.

66. van der Schaft TL, de Bruijn WC, Mooy CM, de Jong PT. Basal laminar deposit in the aging peripheral human retina. Graefes Arch Clin Exp. 1993;231:470-5.

67. Loeffler K, Lee W. Terminology of sub-RPE deposits: do we all speak the same language? Br J Ophthalmol. 1998;82: 1104-5.

68. Sarks SH. Council Lecture. Drusen and their relationship to senile macular degeneration. Aust J Ophthalmol. 1980;8: 117-30.

69. Wang Q, Chappell RJ, Klein R, Eisner A, Klein BE, Jensen SC, et al. Pattern of age-related maculopathy in the macular area. The Beaver Dam Eye Study. Invest Ophthalmol Vis Sci. 1996;37: 2234-42.

70. Wang JJ, Rochtchina E, Lee AJ, Chia EM, Smith W, Cumming $\mathrm{RG}$, et al. Ten-year incidence and progression of age-related maculopathy: the blue Mountains Eye Study. Ophthalmology 2007;114:92-98.

71. Darzins P, Mitchell P, Heller RF. Sun exposure and age-related macular degeneration. An Australian case-control study. Ophthalmology. 1997;104:770-6.

72. Delcourt C, Cougnard-Grégoire A, Boniol M, Carrière I, Doré JF, Delyfer MN, et al. Lifetime exposure to ambient ultraviolet radiation and the risk for cataract extraction and age-related macular degeneration: the Alienor Study. Invest Ophthalmol Vis Sci. 2014;55:7619-27.

73. Marin-Castano ME, Csaky KG, Cousins SW. Nonlethal oxidant injury to human retinal pigment epithelium cells causes cell membrane blebbing but decreased MMP-2 activity. Invest Ophthalmol Vis Sci. 2005;46:3331-40.

74. Davis MD, Gangnon RE, Lee LY, Hubbard LD, Klein B, Klein $\mathrm{R}$ et al. The Age-Related Eye Disease Study severity scale for 
age-related macular degeneration: AREDS report No. 17. Arch Ophthalmol. 2005;123:1484-98.

75. Curcio CA. Soft drusen in age-related macular degeneration: biology and targeting via the oil spill strategies. Invest Ophthalmol Vis Sci. 2018;59:Amd160-81.

76. Ugarte M, Osborne NN. Recent advances in the understanding of the role of zinc in ocular tissues. Metallomics. 2014;6:189-200.

77. Lengyel I, Peto T. Cure or cause: opposing roles for zinc in agerelated macular degeneration. Expert Rev Ophthalmol. 2018;3: $1-4$.

78. Lengyel I, Flinn JM, Petô T, Linkous DH, Cano K, Bird AC, et al. High concentration of zinc in sub-retinal pigment epithelial deposits. Exp Eye Res. 2007;84:772-80.

79. van Kuijk FJ, McPherson SW, Roehrich H. Enhanced detection of sub-retinal pigment epithelial cell layer deposits in human and murine tissue: imaging zinc as a biomarker for age-related macular degeneration (An American Ophthalmological Society Thesis). Trans Am Ophthalmol Soc. 2017;115:T3.

80. Lengyel I, Tufail A, Al Hosaini H, Luthert P, Bird AC, Jeffery G. Association of drusen deposition with choroidal intercapillary pillars in the aging human eye. Invest Ophthalmol Vis Sci. 2004;45:2886-92.

81. Ebrahimi KB, Handa JT. Lipids, lipoproteins, and age-related macular degeneration. J Lipids. 2011;2011:802059.

82. Apte RS. Targeting tissue lipids in age-related macular degeneration. EBioMedicine. 2016;5:26-27.

83. Mullins RF, Schoo DP, Sohn EH, Flamme-Wiese MJ, Workamelahu G, Johnston RM, et al. The membrane attack complex in aging human choriocapillaris: relationship to macular degeneration and choroidal thinning. Am J Pathol. 2014;184: 3142-53.

84. Wang L, Clark ME, Crossman DK, Kojima K, Messinger JD, Mobley JA, et al. Abundant lipid and protein components of drusen. PLoS ONE. 2010;5:e10329.

85. Fernandez-Godino R, Pierce EA, Garland DL. Extracellular matrix alterations and deposit formation in AMD. Adv Exp Med Biol. 2016;854:53-8.

86. Aisenbrey S, Zhang M, Bacher D, Yee J, Brunken WJ, Hunter DD. Retinal pigment epithelial cells synthesize laminins, including laminin 5, and adhere to them through alpha3- and alpha6-containing integrins. Invest Ophthalmol Vis Sci. 2006;47:5537-44.

87. Hageman GS, Mullins RF, Russell SR, Johnson LV, Anderson $\mathrm{DH}$. Vitronectin is a constituent of ocular drusen and the vitronectin gene is expressed in human retinal pigmented epithelial cells. FASEB J. 1999;13:477-84.

88. Johnson LV, Ozaki S, Staples MK, Erickson PA, Anderson DH. A potential role for immune complex pathogenesis in drusen formation. Exp Eye Res. 2000;70:441-9.

89. Mullins RF, Russell SR, Anderson DH, Hageman GS. Drusen associated with aging and age-related macular degeneration contain proteins common to extracellular deposits associated with atherosclerosis, elastosis, amyloidosis, and dense deposit disease. FASEB J. 2000;14:835-46.

90. Crabb JW. The proteomics of drusen. Cold Spring Harb Perspect Med. 2014;4:a017194.

91. Crabb JW, Miyagi M, Gu X, Shadrach K, West KA, Sakaguchi $\mathrm{H}$, et al. Drusen proteome analysis: an approach to the etiology of age-related macular degeneration. Proc Natl Acad Sci U S A. 2002;99:14682-7.

92. Umeda S, Suzuki MT, Okamoto H, Ono F, Mizota A, Terao K, et al. Molecular composition of drusen and possible involvement of anti-retinal autoimmunity in two different forms of macular degeneration in cynomolgus monkey (Macaca fascicularis). FASEB J. 2005;19:1683-5.
93. Hirata C, Nakano K, Nakamura N, Kitagawa Y, Shigeta H, Hasegawa G, et al. Advanced glycation end products induce expression of vascular endothelial growth factor by retinal Muller cells. Biochem Biophys Res Commun. 1997;236:712-5.

94. Pauleikhoff D, Harper CA, Marshall J, Bird AC. Aging changes in Bruch's membrane. A histochemical and morphologic study. Ophthalmology. 1990;97:171-8.

95. Curcio CA, Millican CL, Bailey T, Kruth HS. Accumulation of cholesterol with age in human Bruch's membrane. Invest Ophthalmol Vis Sci. 2001;42:265-74.

96. Haimovici R, Gantz DL, Rumelt S, Freddo TF, Small DM. The lipid composition of drusen, Bruch's membrane, and sclera by hot stage polarizing light microscopy. Invest Ophthalmol Vis Sci. 2001;42:1592-9.

97. John Wiley and Sons. Lipid histochemistry. In: Techniques of biochemical and biophysical morphology. New York: John Wiley and Sons; 1975.

98. Cano M, Fijalkowski N, Kondo N, Dike S, Handa J. Advanced glycation endproduct changes to Bruch's membrane promotes lipoprotein retention by lipoprotein lipase. Am J Pathol. 2011;179:850-9.

99. Rudolf M, Curcio CA. Esterified cholesterol is highly localized to Bruch's membrane, as revealed by lipid histochemistry in wholemounts of human choroid. J Histochem Cytochem. 2009; 57:731-9.

100. Wolter JR, Falls HF. Bilateral confluent drusen. Arch Ophthalmol. 1962;68:219-26.

101. Wedl C. Grundzüge der pathologischen Histologie. Vienna. 1854.

102. Curcio CA, Presley JB, Malek G, Medeiros NE, Avery DV, Kruth HS. Esterified and unesterified cholesterol in drusen and basal deposits of eyes with age-related maculopathy. Exp Eye Res. 2005;81:731-41.

103. Pikuleva IA, Curcio CA. Cholesterol in the retina: the best is yet to come. Prog Retin Eye Res. 2014;41:64-89.

104. Sene A, Apte RS. Eyeballing cholesterol efflux and macrophage function in disease pathogenesis. Trends Endocrinol Metab. 2014;25:107-14.

105. Curcio CA. Antecedents of soft drusen, the specific deposits of age-related macular degeneration, in the biology of human macula. Invest Ophthalmol Vis Sci. 2018;59:Amd182-94.

106. Shen J, He J, Wang F. Association of lipids with age-related macular degeneration. Discov Med. 2016;22:129-45.

107. Malek G, Li CM, Guidry C, Medeiros NE, Curcio CA. Apolipoprotein $\mathrm{B}$ in cholesterol-containing drusen and basal deposits of human eyes with age-related maculopathy. Am J Pathol. 2003;162:413-25.

108. Zweifel SA, Spaide RF, Curcio CA, Malek G, Imamura Y. Reticular pseudodrusen are subretinal drusenoid deposits.Ophthalmology. 2010;117:303-12.e1.

109. Spaide RF. Age-related choroidal atrophy. Am J Ophthalmol. 2009;147:801-10.

110. Curcio CA, Messinger JD, Sloan KR, McGwin G, Medeiros NE, Spaide RF. Subretinal drusenoid deposits in nonneovascular age-related macular degeneration: morphology, prevalence, topography, and biogenesis model. Retina. 2013;33:265-76.

111. Querques G, Querques L, Martinelli D, Massamba N, Coscas G, Soubrane G, et al. Pathologic insights from integrated imaging of reticular pseudodrusen in age-related macular degeneration. Retina. 2011;31:518-26.

112. Neely D, Zarubina AV, Clark ME, Huisingh CE, Jackson GR, Zhang Y, et al. Association between visual function and subretinal drusenoid deposits in normal and early age-related macular degeneration eyes. Retina. 2017;37:1329-36. 
113. Takahashi A, Ooto S, Yamashiro K, Tamura H, Oishi A, Miyata $\mathrm{M}$, et al. Pachychoroid geographic atrophy: clinical and genetic characteristics. Opthalmol Retina. 2018;2:295-305.

114. Hosoda Y, Yoshikawa M, Miyake M, Tabara Y, Ahn J, Woo SJ, et al. CFH and VIPR2 as susceptibility loci in choroidal thickness and pachychoroid disease central serous chorioretinopathy. Proc Natl Acad Sci USA. 2018;115:6261-6.
115. Baek J, Lee JH, Chung BJ, Lee K, Lee WK. Choroidal morphology under pachydrusen. Clin Exp Ophthalmol. 2019;47: 498-504.

116. Kang SW, Lee H, Bae K, Shin JY, Kim SJ, Kim JM. Investigation of precursor lesions of polypoidal choroidal vasculopathy using contralateral eye findings. Graefes Arch Clin Exp. 2017;255:281-91. 